\title{
3-D Unitary ESPRIT: Accurate Attitude Estimation for Unmanned Aerial Vehicles with a
} Hexagon-Shaped ESPAR Array

\author{
Kefei Liu ${ }^{1}$, João Paulo C. L. da Costa $^{2}$, Hing Cheung So ${ }^{1}$, \\ Florian Roemer ${ }^{3}$, Martin Haardt ${ }^{3}$, and Luiz F. de A. Gadêlha ${ }^{2}$ \\ ${ }^{1}$ Department of Electronic Engineering, City University of Hong Kong, Hong Kong, China \\ ${ }^{2}$ Department of Electrical Engineering, University of Brasília, Brazil \\ ${ }^{3}$ Ilmenau University of Technology, Communications Research Laboratory \\ P.O. Box 100565, D-98684 Ilmenau, Germany \\ E-mail: kefeilau@gmail.com,joaopaulo.dacosta@ene.unb.br, hcso@ee.cityu.edu.hk, \\ florian.roemer@tu-ilmenau.de, martin.haardt@tu-ilmenau.de, luizfernandodag@gmail.com
}

\begin{abstract}
Accurate estimation of the attitude of unmanned aerial vehicles (UAVs) is crucial for their control and displacement. Errors in the attitude estimate may misuse the limited battery energy of UAVs or even cause an accident. For attitude estimation, proprioceptive sensors such as inertial measurement units (IMUs) are widely applied, but they are susceptible to inertial guidance error. With antenna arrays currently being installed in UAVs for communication with ground base stations, we can take advantage of the array structure in order to improve the estimates of IMUs via data fusion. In this paper, we therefore propose an attitude estimation system based on a hexagon-shaped 7-element electronically steerable parasitic antenna radiator (ESPAR) array. The ESPAR array is well-suited for installment in the UAVs with broad wings and short bodies. Our proposed solution returns an estimation for the pitch and roll based on the inter-element phase delay estimates of the line-of-sight path of the impinging signal over the antenna array. By exploiting the parallel and centrosymmetric structure in the hexagon-shaped ESPAR array, the 3-dimensional Unitary ESPRIT algorithm is applied for phase delay estimation to achieve high accuracy as well as computational efficiency. We devise an attitude estimation algorithm by exploiting the geometrical relationship between the UAV attitude and the estimated phase delays. An analytical closed-form expression of the attitude estimates are obtained by solving the established simultaneous nonlinear equations. Simulations results show the feasibility of our proposed solution for different signal-to-noise ratio levels as well as multipath scenarios.
\end{abstract}

The work described in this paper was supported by a grant from the Research Grants Council of the Hong Kong Special Administrative Region, China (Project No. CityU 120911) and by the MCT/FINEP through the public announcement CT-AERO - VANT 01/2009. 

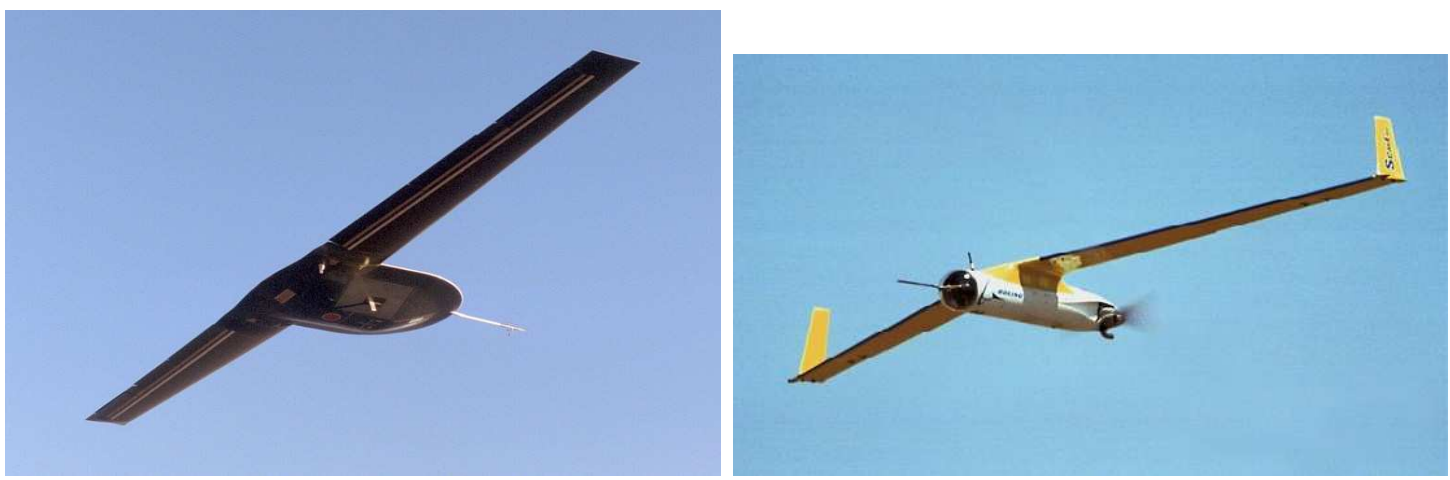

Fig. 1. Darkstar $\mathrm{UAV}^{1}$ (top) and ScanEagle $\mathrm{UAV}^{2}$ (bottom).

\section{Keywords}

unmanned aerial vehicle (UAV), attitude estimation, ESPAR, Unitary ESPRIT

\section{INTRODUCTION}

Accurate attitude estimation for unmanned aerial vehicles (UAVs) is vital to ensure their correct displacement and control. The attitude indication of UAVs is usually conducted by inertial measurement units (IMUs) consisting of proprioceptive sensors such as gyroscopes, magnetometers and accelerometers. However, IMUs are subject to the inertial guidance error, which arises from the accelerometer measurement error and the gyroscope drift error [1], [2].

In our previous papers [1], [3], a 4-element cross-shaped antenna array has been used for UAV attitude estimation, which can be used to improve the estimates of IMUs, e.g., via data fusion. In the cross array, two pairs of antennas is located at both ends of the body and wing. This layout is applicable to UAVs which have relatively long body. However, in cases the UAVs have short bodies [4] (see Figure 1), which is typical for most UAVs, attitude estimation based on cross-shaped antennas suffers a degradation in estimation accuracy.

Recently, the hexagon-shaped 7-element electronically steerable parasitic antenna radiator (ESPAR) array has attracted considerable attention [5]-[7]. With only a single-port output, the ESPAR array has low power consumption, small physical size as well as low manufacturing cost which renders it very attractive for practical use in battery operated devices such as UAVs. From the geometry point of view, the 7-antenna ESPAR array is well-suited for installation in UAVs with broad wings and short bodies. In this paper, we use the ESPAR array as an alternative to the cross-shaped array for UAV attitude estimation. Due to the fast development of ESPAR arrays, they have promising application prospects in UAVs for different purposes, e.g., for radar and communication systems [8] [9] [4]. Therefore, our attitude determination solution may not be subject to the endowment of additional equipment. Note that in the literature [10], [11], other attitude estimation solutions based on GPS receivers and satellite

\footnotetext{
${ }^{1}$ http://www.dreamlandresort.com/black_projects/darkstar.html

${ }^{2}$ http://www.naval-technology.com/projects/scaneagle-uav/scaneagle-uav1.html
} 
communication links are also proposed. However, such solutions require an additional installation of a GPS antenna array in UAVs, which increases their costs and weights.

The first crucial step in the attitude estimation algorithm is to determine the phase delay, whose accuracy directly affects the former performance. By exploiting the parallel and centrosymmetric structure of the hexagon-shaped ESPAR array, we apply the 3-dimensional (3-D) Unitary ESPRIT algorithm [7], [12] for phase delay estimation. The 3-D Unitary ESPRIT enjoys the advantages of a high estimation accuracy and a low computational complexity due to the use of the forward-backward averaging (FBA) technique and a real-valued transformation (RVT).

The remainder of this paper is organized as follows. In Section II we explain the system model for the UAV equipped with the hexagon-shaped 7-element ESPAR antenna array. Our proposed attitude estimation scheme consists of two stages, namely, phase delay estimation and calculation of the pitch and roll, which are presented in Sections III and IV, respectively. In Section V, simulation results are provided to evaluate the performance of the proposed algorithm, and finally, conclusions are drawn in Section VI.

\section{SYSTEM MODEL}

In this section, we present the model of our considered system, which consists of the scenario description, the definition of the attitude angles and data model.

\section{A. Scenario Description}

We define a 3-D coordinate system [1], [3] according to Figure 2 with the base station placed at the origin. A UAV is endowed with an ESPAR array with seven antennas in a flattened hexagonal shape. For the sake of simplicity, we assume that all antennas lie in the same plane and that the wings lie on a straight line perpendicular to the longitudinal axis. $S_{4}$ is situated at the symmetric center of the hexagon (which coincides with the intersection point of the body and wing axes), and the remaining six antennas are symmetrically distributed on the wing: $S_{1}$ and $S_{7}$ are located at the midpoints of the pair of short opposite sides, and $S_{2}, S_{3}, S_{5}$ and $S_{6}$ symmetrically located at the quartile points of the pair of long opposite sides. A GPS sensor is placed at the symmetric center of the UAV array to provide the GPS coordinates of the UAV. Moreover, we assume that the GPS coordinates of the base station are known. From the GPS coordinates of the UAV, the UAV coordinates in the base station coordinate system can be obtained by a coordinate transformation. The length of the long side of the wing, namely, the distance from one wingtip to the other wingtip, is referred to as the wingspan, denoted as $d_{\text {wing, }}$, while the length of its short side is

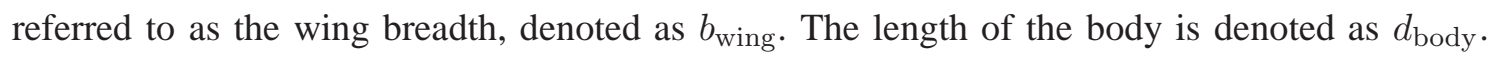

\section{B. Definitions}

In Figure 3, the definitions of the attitude angles for pitch, roll and yaw are shown by establishing the 3-D coordinate system with origin located in the symmetric center of the UAV array and coordinate axes parallel to those of the GPS coordinate systems. The pitch $\varphi \in[-\pi / 2, \pi / 2]$ is defined by the elevation angle between the 


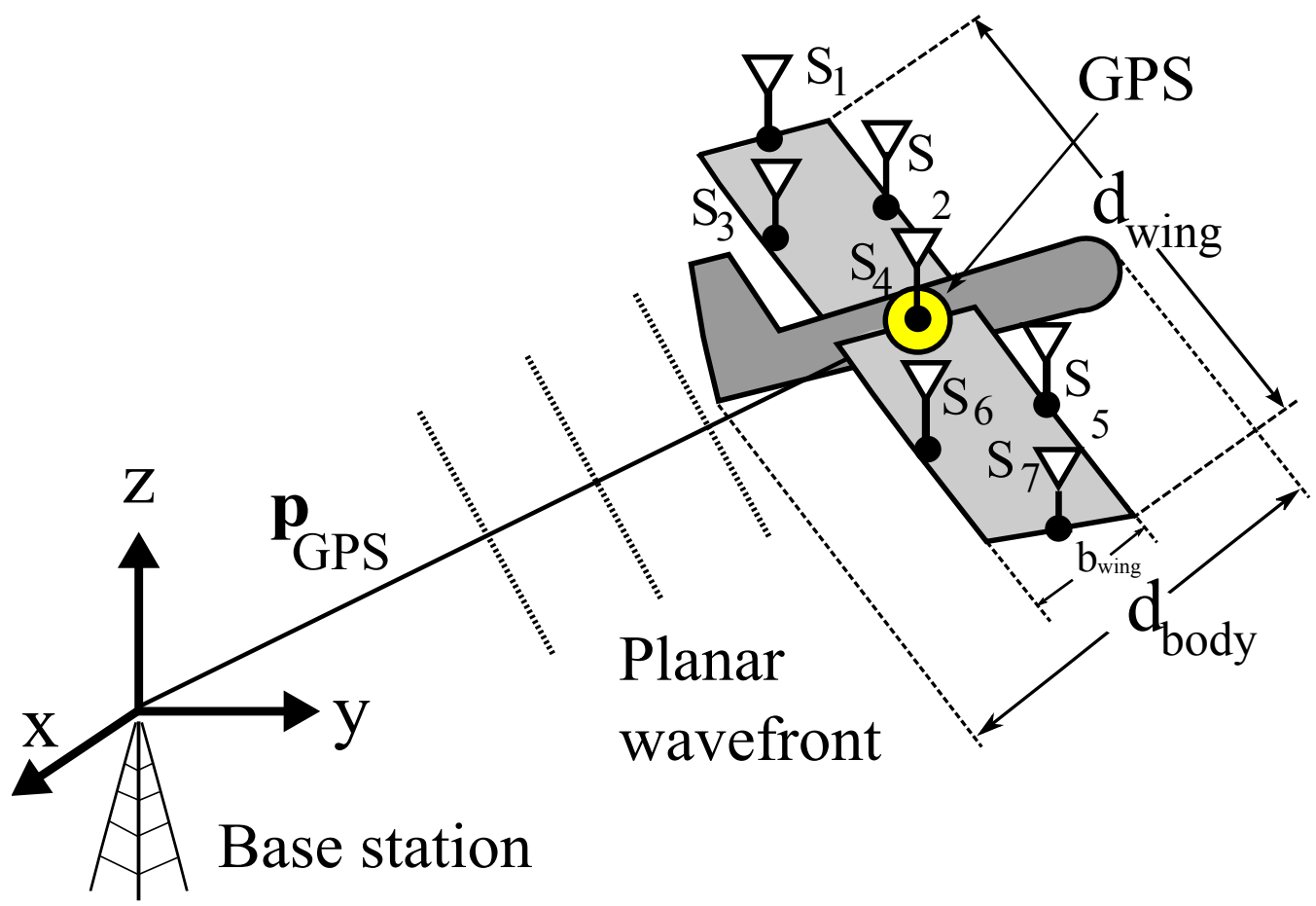

Fig. 2. System arrangement depicting communication link between base station and UAV endowed with an 7-element ESPAR array.

$x-y$ plane and the longitudinal (body) axis of the UAV. The roll $\theta \in(-\pi, \pi]$ is given by the rotation angle of the wings about the body axis. In case $\varphi=0$, the roll $\theta$ is the angle between the $x-y$ plane and the lateral (wing) axis. The azimuth angle, namely, the rotational angle about the $z$ axis, is referred to as yaw. In this paper, the yaw $\psi \in[0,2 \pi)$ is assumed to be known a priori since it is determined in a different way from the pitch and roll [13].

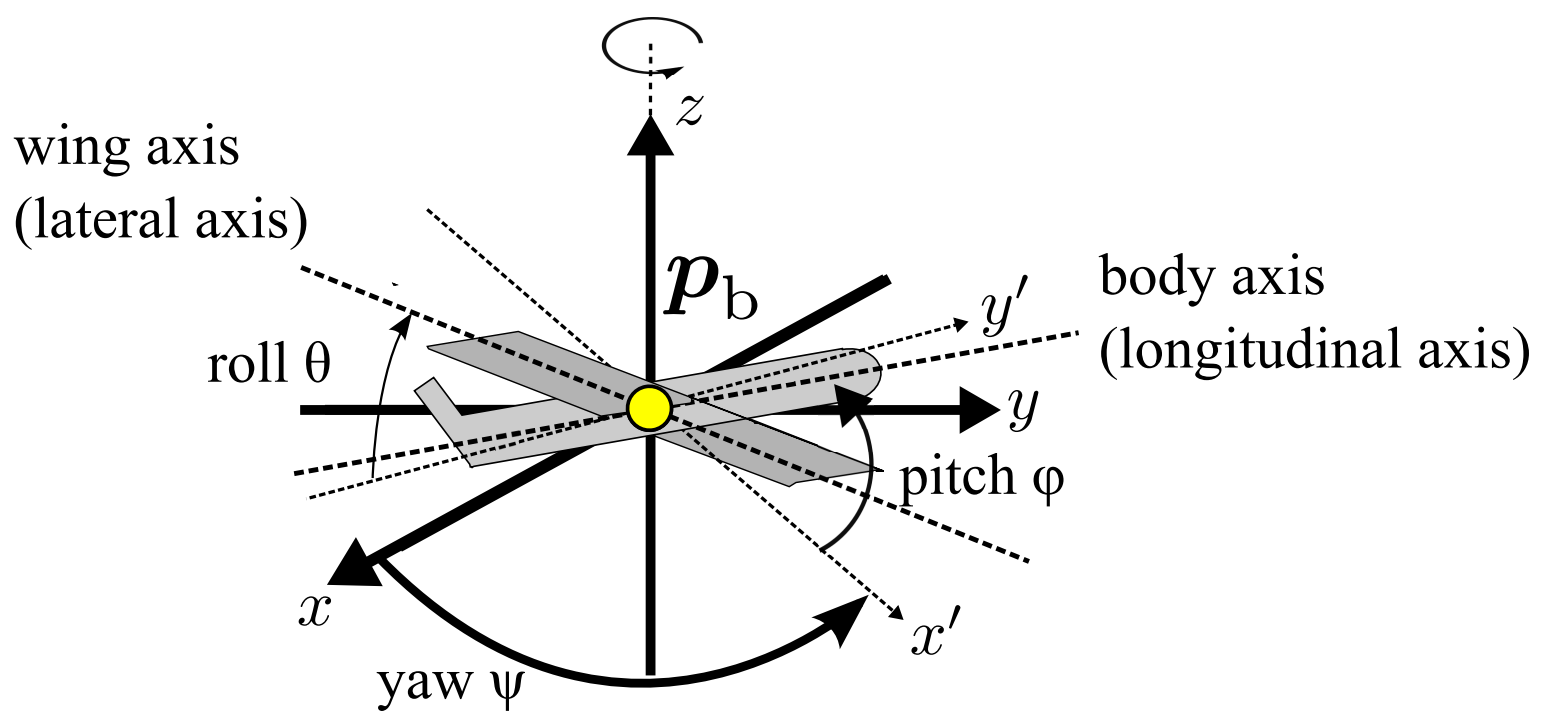

Fig. 3. Definitions of pitch $\varphi$, roll $\theta$ and yaw $\psi$ in 3-D coordinate system. 


\section{Data Model}

Assume that a source is radiating narrowband signals from the far field of the array, i.e., its wavefronts can be considered to be planar at the receiver. In multipath environment, the received signal is a mixture of a line-of-sight (LOS) path and $(K-1)$ non-line-of-sight (NLOS) multipath components. Here we assume that a strong LOS is always present. In ESPAR arrays, there is only one output which is connected to the center antenna, namely, $S_{4}$, while each of the remaining six antennas is loaded with an adjustable reactance through a connected varactor diode. The scalar output is a weighted combination of the outputs of all the elements and the weights can be controlled by appropriate steering of the varactor diodes. To obtain the output of the $m$-th, $m=1,2, \ldots, M$, antenna, we need to apply at least $M$ different sets of reactances where each provides us with a different linear combination of the actual array outputs [6], [7]. Since we know the weights of these linear combinations, we can recover the signal at the passive elements by inverting the weighting.

The base band output of the corresponding decoupled 7-output array can therefore be written as

$$
\boldsymbol{X}=\boldsymbol{A S}+\boldsymbol{Z},
$$

where $\boldsymbol{X} \in \mathbb{C}^{7 \times N}$ is the measurement matrix, $\boldsymbol{A} \in \mathbb{C}^{7 \times K}$ denotes the array steering matrix which consists of $K$ array steering vectors $\boldsymbol{a}_{1}, \ldots, \boldsymbol{a}_{K}, \boldsymbol{S} \in \mathbb{C}^{K \times N}$ contains the $N$ symbols from all $K$ multipaths, with an average signal power of $\sigma_{i}^{2}$ in the $i$-th multipath, and $Z \in \mathbb{C}^{7 \times N}$ is the noise matrix collecting the additive noise samples which are assumed to be mutually uncorrelated ${ }^{3}$ and Gaussian distributed with zero mean and variance of $\sigma_{z}^{2}$.

Our goal in this paper is to estimate the pitch $\varphi$ and the roll $\theta$ using the hexagon-shaped ESPAR antenna array. Our proposed attitude estimation scheme consists of two stages. First in Section III, the inter-element differences in phase delays (IDPhDs) of the LOS path between the antenna pair along the three symmetry axes in the ESPAR array are estimated using 3-D Unitary ESPRIT parameter estimation algorithm. Second, in Section IV, based on the geometrical relationship between the UAV attitude and IDPhDs, a set of nonlinear equations is established and solved to estimate the attitude pitch and roll.

\section{3-D UnitARy ESPRIT FOR Phase Delay Estimation}

To begin with, let us first investigate the IDPhD associated to a single planar wavefront. The IDPhDs along the three symmetry axes of the hexagon are defined as

$$
\begin{aligned}
& \mu \triangleq \phi_{4}-\phi_{1}, \\
& \nu \triangleq \phi_{4}-\phi_{3}, \\
& \omega \triangleq \phi_{4}-\phi_{2},
\end{aligned}
$$

where $\phi_{i}, i=1, \ldots, 4$ is the phase delay of the antenna $S_{i}$.

In this section, we discuss the estimation of $\mu, \nu$, and $\omega$ of the LOS path via 3-D Unitary ESPRIT [7].

${ }^{3}$ If the noise is correlated (which may be the case for ESPAR arrays due to the decoupling), prewhitening will be applied as a preprocessing step. 


\section{A. Shift invariance}

Since antennas $S_{i}, i=1,2,3,4$ form a parallelogram, we have

$$
\mu+\omega=\nu .
$$

We can express the array steering vector $\boldsymbol{a}$ in terms of the spatial frequencies as

$$
\boldsymbol{a}(\mu, \nu, \omega)=\left[e^{-j \mu}, e^{j \omega}, e^{-j \nu}, 1, e^{j \nu}, e^{-j \omega}, e^{j \mu}\right]^{T} .
$$
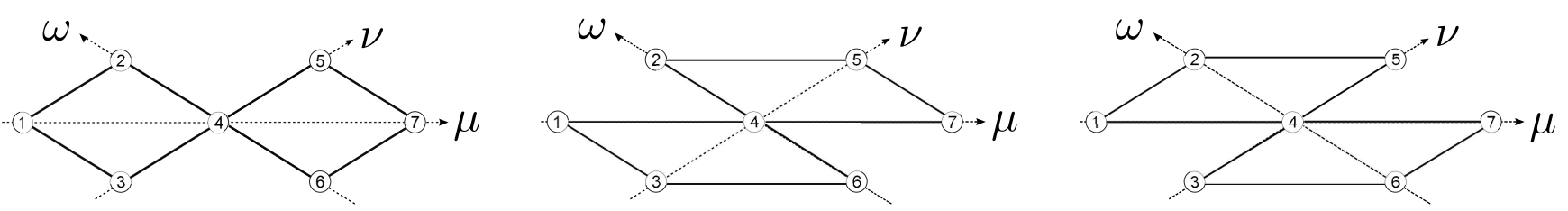

Fig. 4. The ESPAR array and its invariances.

Note from (5)-(6) and Figure 4 that the array is shift invariant along all three axes we have defined. In each case, $m=4$ out of $M=7$ elements belong to one subarray, and the center element $S_{4}$ is the only one common to all of them. In order to apply ESPRIT methods, we have to define selection matrices that select four out of seven elements belonging to the desired subarrays. Let us consider the $\mu$-direction first. In this case, $S_{1}$ is mapped to $S_{4}$, $S_{2}$ to $S_{5}$ and so on. Therefore, the selection matrices in $\mu$-direction are defined as [7]

$$
\begin{aligned}
\boldsymbol{J}_{\mu, 1}= & {\left[\begin{array}{lllllll}
1 & 0 & 0 & 0 & 0 & 0 & 0 \\
0 & 1 & 0 & 0 & 0 & 0 & 0 \\
0 & 0 & 1 & 0 & 0 & 0 & 0 \\
0 & 0 & 0 & 1 & 0 & 0 & 0
\end{array}\right] } \\
\boldsymbol{J}_{\mu, 2}= & {\left[\begin{array}{lllllll}
0 & 0 & 0 & 1 & 0 & 0 & 0 \\
0 & 0 & 0 & 0 & 1 & 0 & 0 \\
0 & 0 & 0 & 0 & 0 & 1 & 0 \\
0 & 0 & 0 & 0 & 0 & 0 & 1
\end{array}\right] . }
\end{aligned}
$$


Likewise, applying the shift invariant conditions to the other two directions yielding the following results [7]

$$
\begin{aligned}
\boldsymbol{J}_{\nu, 1}= & {\left[\begin{array}{lllllll}
1 & 0 & 0 & 0 & 0 & 0 & 0 \\
0 & 0 & 1 & 0 & 0 & 0 & 0 \\
0 & 0 & 0 & 1 & 0 & 0 & 0 \\
0 & 0 & 0 & 0 & 0 & 1 & 0
\end{array}\right] } \\
\boldsymbol{J}_{\nu, 2}= & {\left[\begin{array}{lllllll}
0 & 1 & 0 & 0 & 0 & 0 & 0 \\
0 & 0 & 0 & 1 & 0 & 0 & 0 \\
0 & 0 & 0 & 0 & 1 & 0 & 0 \\
0 & 0 & 0 & 0 & 0 & 0 & 1
\end{array}\right] } \\
\boldsymbol{J}_{\omega, 1}= & {\left[\begin{array}{lllllll}
0 & 0 & 1 & 0 & 0 & 0 & 0 \\
0 & 0 & 0 & 1 & 0 & 0 & 0 \\
0 & 0 & 0 & 0 & 0 & 1 & 0 \\
0 & 0 & 0 & 0 & 0 & 0 & 1
\end{array}\right] } \\
\boldsymbol{J}_{\omega, 2}= & {\left[\begin{array}{lllllll}
1 & 0 & 0 & 0 & 0 & 0 & 0 \\
0 & 1 & 0 & 0 & 0 & 0 & 0 \\
0 & 0 & 0 & 1 & 0 & 0 & 0 \\
0 & 0 & 0 & 0 & 1 & 0 & 0
\end{array}\right] . }
\end{aligned}
$$

\section{B. 3-D Unitary ESPRIT}

In order to estimate the spatial frequencies along three directions jointly, we use the 3-D Unitary ESPRIT algorithm [7]. The ESPRIT-type algorithms use the signal subspace to estimate the spatial frequencies. Therefore, the first step is to compute the eigenvalue decomposition of the sample covariance matrix (SCM)

$$
\hat{\boldsymbol{R}}_{\mathrm{xx}}=\frac{1}{N} \boldsymbol{X} \boldsymbol{X}^{\mathrm{H}} \in \mathbb{C}^{M \times M},
$$

where ${ }^{\mathrm{H}}$ represents the Hermitian transpose of a matrix. In principle, the $K$ eigenvectors associated to the $K$ eigenvalues with the greatest power are assumed to form the signal subspace $\boldsymbol{U}_{s}$, and the $(M-K)$ eigenvectors related to the $(M-K)$ eigenvalues with smallest power are assumed to be the noise subspace. Here, we assume that the line-of-sight (LOS) path is always present and it has higher power than all non-line-of-sight (NLOS) paths, which is valid in nominal conditions. That is to say, the NLOS paths are treated as interference. In doing so, we only choose $K=1$.

The spatial frequencies are computed as

$$
\eta=\arg \left(\left(\boldsymbol{J}_{\eta, 1} \boldsymbol{u}_{s}\right)^{\mathrm{H}} \boldsymbol{J}_{\eta, 2} \boldsymbol{u}_{s}\right), \quad \eta \in\{\mu, \nu, \omega\}
$$

where $\boldsymbol{u}_{s}$ is the dominant eigenvector of the SCMthat corresponds to the largest eigenvalue. 
This solution is the standard ESPRIT algorithm. In order to improve the estimation accuracy and the computational efficiency, we use 3-D Unitary ESPRIT [7], [14]. By applying FBA on the measurement data, we obtain a centroHermitian matrix which is then mapped to a real-valued matrix by [15]

$$
\mathcal{T}(\boldsymbol{X})=\boldsymbol{Q}_{M}^{\mathrm{H}}\left[\boldsymbol{X}, \boldsymbol{\Pi}_{M} \boldsymbol{X}^{*} \boldsymbol{\Pi}_{N}\right] \boldsymbol{Q}_{2 N},
$$

where $^{*}$ denotes the complex conjugation, $\boldsymbol{\Pi}_{p}$ is the $p \times p$ exchange matrix with ones on its antidiagonal and zeros elsewhere, and $\boldsymbol{Q}_{p} \in \mathbb{C}^{p \times p}$ is a unitary left- $\boldsymbol{\Pi}$-real matrix which satisfies $\boldsymbol{\Pi}_{p} \boldsymbol{Q}_{p}^{*}=\boldsymbol{Q}_{p}$.

We get the following modified shift invariance equations

$$
\begin{aligned}
\boldsymbol{K}_{\mu, 1} \boldsymbol{e}_{s} \lambda_{\mu} & \approx \boldsymbol{K}_{\mu, 2} \boldsymbol{e}_{s} \\
\boldsymbol{K}_{\nu, 1} \boldsymbol{e}_{s} \lambda_{\nu} & \approx \boldsymbol{K}_{\nu, 2} \boldsymbol{e}_{s} \\
\boldsymbol{K}_{\omega, 1} \boldsymbol{e}_{s} \lambda_{\omega} & \approx \boldsymbol{K}_{\omega, 2} \boldsymbol{e}_{s}
\end{aligned}
$$

where $e_{s} \in \mathbb{R}^{M \times 1}$ is the dominant eigenvector obtained from the transformed real-valued data matrix in (15), and the new selection matrices $\boldsymbol{K}_{\eta, i}, \eta \in\{\mu, \nu, \omega\}, i=1,2$, are obtained from $\boldsymbol{J}_{\eta, i}$ by

$$
\begin{aligned}
\boldsymbol{K}_{\eta, 1} & =2 \cdot \operatorname{Re}\left\{\boldsymbol{Q}_{m}^{\mathrm{H}} \boldsymbol{J}_{\eta, 2} \boldsymbol{Q}_{M}\right\} \\
\boldsymbol{K}_{\eta, 2} & =2 \cdot \operatorname{Im}\left\{\boldsymbol{Q}_{m}^{\mathrm{H}} \boldsymbol{J}_{\eta, 2} \boldsymbol{Q}_{M}\right\},
\end{aligned}
$$

where $\operatorname{Re}\{\cdot\}$ and $\operatorname{Im}\{\cdot\}$ represents the real and imaginary part of a complex matrix, respectively.

Finally, the spatial frequencies are obtained as

$$
\begin{aligned}
& \hat{\mu}=2 \cdot \tan ^{-1}\left(\lambda_{\mu}\right), \\
& \hat{\nu}=2 \cdot \tan ^{-1}\left(\lambda_{\nu}\right), \\
& \hat{\omega}=2 \cdot \tan ^{-1}\left(\lambda_{\omega}\right) .
\end{aligned}
$$

\section{Attitude Determination Algorithm}

We assume there is a pair of dummy antennas $S_{8}$ and $S_{9}$ along the body axes which lie on the extension of the wing antennas, as shown in Figure 5. These dummy antennas and other antennas form the parallelogram which is centered around $S_{4}$.

The estimated phase delay differences of the LOS path between the wing antennas $\left(S_{1}, S_{4}\right)$ and body antennas $\left(S_{4}, S_{8}\right)$ are obtained as

$$
\begin{aligned}
& \hat{\mu}_{\text {wing }}=\hat{\mu}^{(\mathrm{LOS})} \\
& \hat{\mu}_{\text {body }}=\hat{\nu}^{(\mathrm{LOS})}+\hat{\omega}^{(\mathrm{LOS})} .
\end{aligned}
$$

The main idea of the algorithm is to determine the coordinates for the left wing antenna $S_{1}$ and dummy nose antenna $S_{8}$ as a function of the unknown pitch and roll, and establish a system of simultaneous nonlinear equations based on the array geometry and estimated phase delays $\hat{\mu}_{\text {wing }}$ and $\hat{\mu}_{\text {body }}$ [1], [3]. 


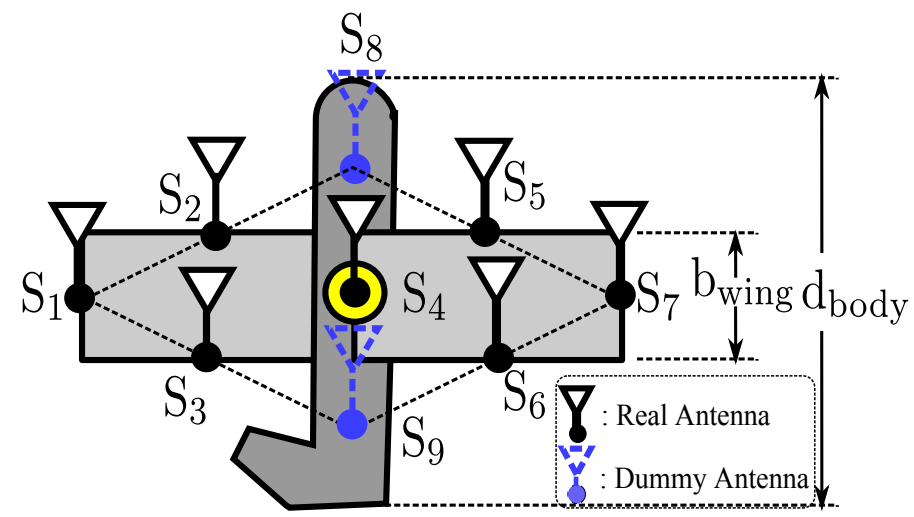

Fig. 5. Positions of the pair of dummy antennas $\left(S_{8}, S_{9}\right)$ in the ESPAR array.

a)
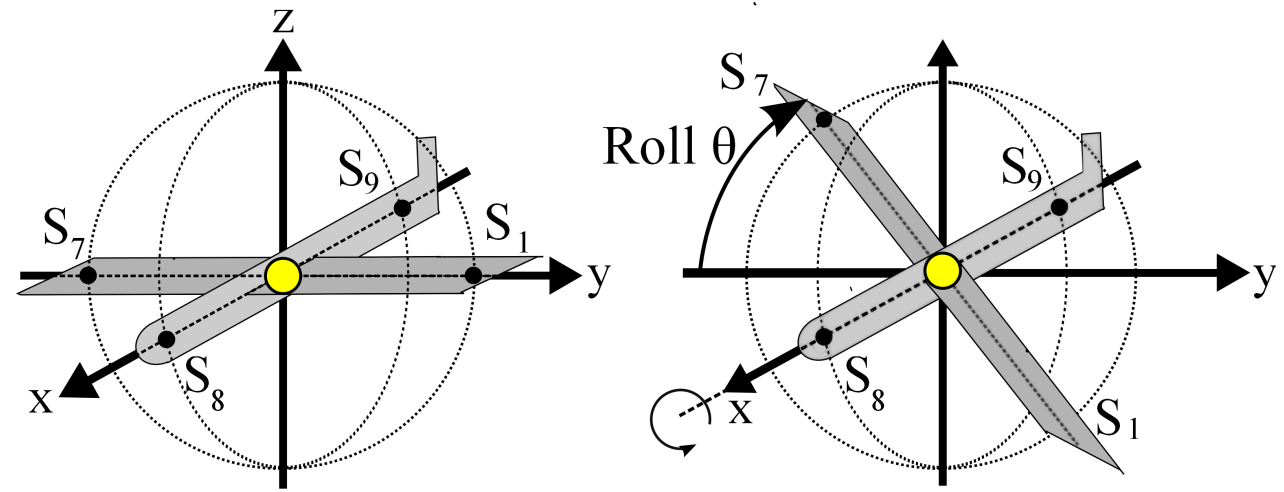

c)
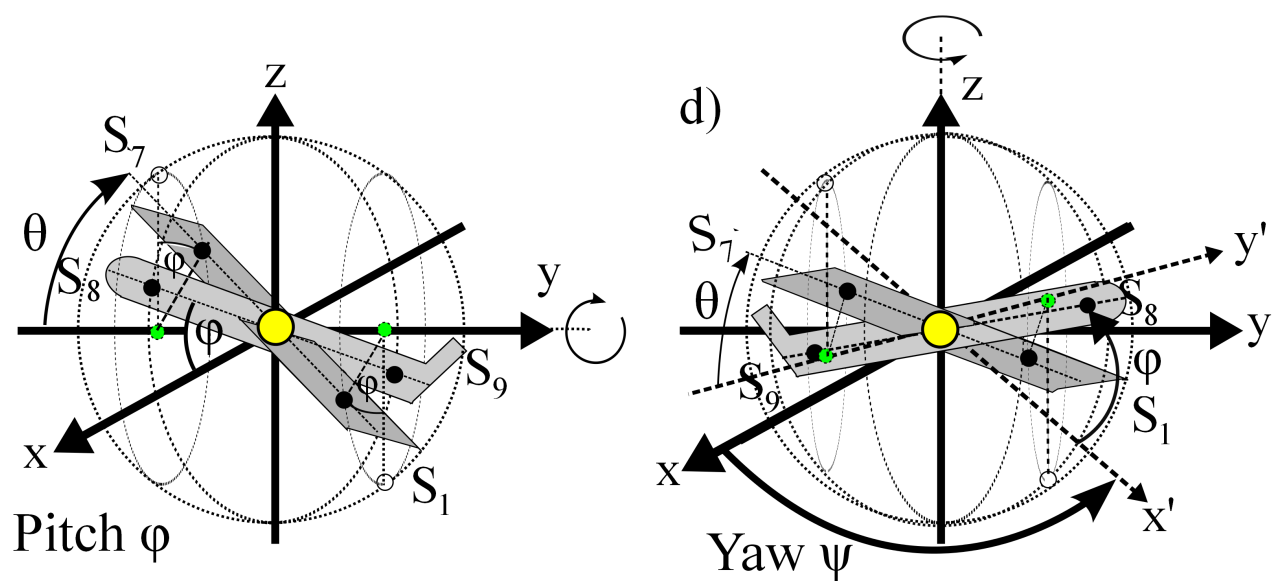

Fig. 6. Illustration of antenna positions in $x-y-z$ system [1], [3]: a) basic configuration $(0,0,0)$; b) configuration with added roll $(0, \theta, 0)$; c) configuration with added pitch $(\varphi, \theta, 0)$; d) configuration with added yaw movement $(\varphi, \theta, \psi)$

Figure 6 describes how a specified attitude $(\varphi, \theta, \psi)$ of a UAV is formed [1], [3]. Initially, the body of the UAV is identical to the $x$ axis, and the wing coincides with the $y$ axis (Figure 6a). When evoking a roll movement, the wing antennas $S_{1}$ and $S_{7}$ rotate about the $x$ axis in the $y-z$ plane, as depicted in Figure $6 \mathrm{~b}$. It is followed by successive pitch and yaw movements which correspond to the rotations of all antennas about the $y$ and $z$ axis, respectively, as described in Figures $6 \mathrm{c}$ and $6 \mathrm{~d}$. Note that as a consequence of the chosen rotation order (roll - pitch 
- yaw), all the rotations are about the axes.

The coordinates for the dummy nose antenna $S_{8}$ are

$$
\boldsymbol{p}_{8}^{\prime}(\varphi, \psi)=b_{\text {wing }} \boldsymbol{R}_{z}(\psi)\left[\begin{array}{c}
0 \\
\cos \varphi \\
\sin \varphi
\end{array}\right]
$$

where a yaw motion $\psi$ is taken into account by the counter-clockwise rotation matrix

$$
\boldsymbol{R}_{z}(\psi)=\left(\begin{array}{ccc}
\cos \psi & -\sin \psi & 0 \\
\sin \psi & \cos \psi & 0 \\
0 & 0 & 1
\end{array}\right) .
$$

Next, we take into account the roll movement. The left wing antenna $S_{1}$ lies on a circle in the $x-z$ plane which experiences two subsequent rotations about the $x$ axis and $z$ axis (Figure 6c-d). The coordinates of the left wing antenna are therefore given by

$$
\boldsymbol{p}_{1}^{\prime}(\varphi, \theta, \psi)=\frac{1}{2} d_{\text {wing }} \boldsymbol{R}_{z}(\psi) \boldsymbol{R}_{x}(\varphi)\left[\begin{array}{c}
\cos \theta \\
0 \\
\sin \theta
\end{array}\right]
$$

where $\boldsymbol{R}_{x}(\varphi)$ represents the rotation about the $x$ axis by an angle of pitch $\varphi$

$$
\boldsymbol{R}_{x}(\varphi)=\left(\begin{array}{ccc}
1 & 0 & 0 \\
0 & \cos \varphi & -\sin \varphi \\
0 & \sin \varphi & \cos \varphi
\end{array}\right) .
$$

Finally, the antenna positions relative to the base station $\boldsymbol{p}_{i}, i=1,8$, are obtained by adding the UAV coordinates $\boldsymbol{p}_{\mathrm{b}}$ to the local antenna coordinates $\boldsymbol{p}_{i}^{\prime}$

$$
\begin{aligned}
\boldsymbol{p}_{1}(\varphi, \theta, \psi) & =\boldsymbol{p}_{1}^{\prime}(\varphi, \theta, \psi)+\boldsymbol{p}_{\mathrm{b}} \\
\boldsymbol{p}_{8}(\varphi, \psi) & =\boldsymbol{p}_{8}^{\prime}(\varphi, \psi)+\boldsymbol{p}_{\mathrm{b}} .
\end{aligned}
$$

Using the phase difference between the body antennas $\hat{\omega}_{\text {body }}$ calculated in Section III by 3-D Unitary ESPRIT, we set up the following equation

$$
\left\|\boldsymbol{p}_{8}(\varphi, \psi)\right\|-\left\|\boldsymbol{p}_{\mathrm{b}}\right\|=-\frac{\lambda}{2 \pi} \hat{\mu}_{\text {body }}
$$

where $\lambda \hat{\mu}_{\text {body }} /(2 \pi)$ is the estimated propagation distance difference of the antenna pair $\left(S_{1}, S_{8}\right)$.

Solving (30) yields (see Appendix A)

$$
\hat{\varphi}=\sin ^{-1}\left(\frac{-\lambda / \pi \cdot \hat{\mu}_{\text {body }}\left\|\boldsymbol{p}_{\mathrm{b}}\right\|+\lambda^{2} /\left(4 \pi^{2}\right) \cdot \hat{\mu}_{\text {body }}^{2}-b_{\text {wing }}^{2}}{2 b_{\text {wing }} \sqrt{\left(x_{\mathrm{b}} \cos \psi-y_{\mathrm{b}} \sin \psi\right)^{2}+z_{\mathrm{b}}^{2}}}\right)-\alpha,
$$

Once an estimate for the pitch $\hat{\varphi}$ is obtained, we compute the roll $\theta$ using $\hat{\mu}_{\text {wing }}$ in a similar way

$$
\left\|\boldsymbol{p}_{1}(\hat{\varphi}, \theta, \psi)\right\|-\left\|\boldsymbol{p}_{\mathrm{b}}\right\|=\frac{\lambda}{2 \pi} \hat{\mu}_{\text {wing }} .
$$


A pair of supplementary solutions are obtained from solving (32) (see Appendix A)

$$
\hat{\theta}_{1}=\sin ^{-1}\left(\frac{\lambda / \pi \cdot \hat{\mu}_{\text {wing }}\left\|\boldsymbol{p}_{\mathrm{b}}\right\|+\lambda^{2} /\left(4 \pi^{2}\right) \cdot \hat{\mu}_{\text {wing }}^{2}-d_{\text {wing }}^{2} / 4}{d_{\text {wing }} \sqrt{\left(x_{\mathrm{b}} \cos \psi+y_{\mathrm{b}} \sin \psi\right)^{2}+\left(x_{\mathrm{b}} \sin \psi \sin \hat{\varphi}-y_{\mathrm{b}} \cos \psi \sin \hat{\varphi}+z_{\mathrm{b}} \cos \hat{\varphi}\right)^{2}}}\right)-\beta,
$$

and

$$
\hat{\theta}_{2}=\pi-\sin ^{-1}\left(\frac{\lambda / \pi \cdot \hat{\mu}_{\text {wing }}\left\|\boldsymbol{p}_{\mathrm{b}}\right\|+\lambda^{2} /\left(4 \pi^{2}\right) \cdot \hat{\mu}_{\mathrm{wing}}^{2}-d_{\mathrm{wing}}^{2} / 4}{d_{\mathrm{wing}} \sqrt{\left(x_{\mathrm{b}} \cos \psi+y_{\mathrm{b}} \sin \psi\right)^{2}+\left(x_{\mathrm{b}} \sin \psi \sin \hat{\varphi}-y_{\mathrm{b}} \cos \psi \sin \hat{\varphi}+z_{\mathrm{b}} \cos \hat{\varphi}\right)^{2}}}\right)-\beta,
$$

where

$$
\beta=\tan ^{-1}\left(\frac{x_{\mathrm{b}} \cos \psi+y_{\mathrm{b}} \sin \psi}{x_{\mathrm{b}} \sin \psi \sin \hat{\varphi}-y_{\mathrm{b}} \cos \psi \sin \hat{\varphi}+z_{\mathrm{b}} \cos \hat{\varphi}}\right) .
$$

We cannot decide which one of this pair of supplementary angles is correct from the algorithm itself. This is the inherent uncertainty of the proposed algorithm. In order to have a unique solution, we assume that a UAV cannot be flying upside down, which is valid in nominal conditions, implying that $\theta \in[-\pi / 2, \pi / 2]$. Thereby, only the first solution, namely, (33) is chosen.

\section{Simulation Results}

We evaluate our proposed algorithm by means of Monte Carlo simulations. The data is generated based on (1), where the signal samples are complex sinusoidal with unit amplitude and frequency of $30 \mathrm{MHz}$. The UAV is located sufficiently far from the base station (1000 m here) such that the assumption on planar waves approximately holds. We consider a scenario where the UAV has a wingspan of $d_{\text {wing }}=1.2 \mathrm{~m}$ and a wing breadth of $b_{\text {wing }}=0.39 \mathrm{~m}$. 1000 independent Monte Carlo runs are conducted. In each run, the pitch $\varphi$ and roll $\theta$ are uniformly generated in the interval $[-\pi / 2, \pi / 2]$, the yaw is uniformly generated in $[0,2 \pi))$ and is assumed to be known. The closed-form solutions of (31) and (33)(34) are used as the pitch and roll estimates. The root mean square error (RMSE) of the estimated pitch and roll defined as

$$
\begin{aligned}
\operatorname{RMSE}(\hat{\varphi}) & =\sqrt{\frac{1}{2 L} \sum_{l=1}^{L}\left(\hat{\varphi}_{l}-\varphi\right)^{2}}, \\
\operatorname{RMSE}(\hat{\theta}) & =\sqrt{\frac{1}{2 L} \sum_{l=1}^{L}\left(\hat{\theta}_{l}-\theta\right)^{2}},
\end{aligned}
$$

where $L$ is the number of independent Monte Carlo runs, is used as the performance measure. For comparison, the cross-shaped array combined with 2-D ESPRIT proposed in [3] is used as the benchmark.

\section{A. Performance Comparison: Hexagon-Shaped Array versus Cross-Shaped Array [1], [3]}

Denote the body-length-to-wing-breadth ratio as $r=d_{\text {body }} / b_{\text {wing }}$. We compare the attitude estimation performance of our proposed hexagon-shaped array combined with 3-D Unitary ESPRIT and the cross-shaped array combined with 2-D ESPRIT under various $r$. Here the body length varies to get different $r$. 
First, we consider the scenario where only the LOS path is present $(K=1)$. The signal-to-noise ratio (SNR) in $\mathrm{dB}$ is defined as

$$
\mathrm{SNR}=10 \log _{10}\left(\frac{\sigma_{1}^{2}}{\sigma_{\mathrm{z}}^{2}}\right) .
$$

The noise samples are modeled as complex-valued i.i.d. zero mean white Gaussian processes, whose power is scaled to produce different SNRs.

In Figures 7 and 8, we respectively plot the RMSEs of pitch and roll estimates, measured in degrees, versus SNR for two attitude estimation schemes. Note that when the UAV has a short body and a broad wing such that $r$ is small $(r<2)$, the hexagon-shaped array in conjunction with 3-D Unitary ESPRIT outperforms the cross-shaped array combined with 2-D ESPRIT in both pitch and roll estimation. And the improvement is more significant with the dicrease of $r$. And for $r=2$, they have almost the same performance. On the other hand,when the UAV has a long body and a narrow wing such that $r$ is large $(r>2)$, the hexagon-shaped array combined with 3-D Unitary ESPRIT is outperformed by the cross-shaped array combined with 2-D ESPRIT in terms of pitch estimation. Nevertheless, it is slightly superior to the latter in terms of roll estimation for $r \leq 4$. This shows that the hexagon-shaped array combined with 3-D Unitary ESPRIT has more advantages in roll estimation. Note also that the pitch estimation accuracy is more affected by the variation of the body length.

An intuitive explanation behind such an observation is an aperture argument. For small $r<2$, the cross-shaped antenna is confined to the body length whereas the hexagon-shaped array extends beyond it via the dummy antennas $S_{8}$ and $S_{9}$ and hence has a larger aperture. On the other hand, for large $r>2$, the cross-shaped antenna simply spans a larger area and hence has a larger aperture that is why it yields more accurate estimates. For $r=2$, the cross-shaped antenna and the hexagon-shaped array have equal aperture which results in the same performance.

In Figures 10 and 11, the effect of multipath components is considered in the presence of noise. The LOS-toNLOS power ratio, commonly known as the signal-to-interference power ratio (SIR), is given by

$$
\mathrm{SIR}=10 \log _{10}\left(\frac{\sigma_{1}^{2}}{\sum_{i=2}^{K} \sigma_{i}^{2}}\right),
$$

where we assume that $\sigma_{1}^{2}$ corresponds to the signal power of the LOS path, and $\sigma_{i}^{2}, i=2, \ldots, K$ is the power of the $i$-th multipath component. $K=8$ paths are considered, namely, 1 LOS and 7 NLOS components. For a fixed SIR, the power ratios of different NLOS paths are generated from the squared Gaussian distribution, namely, the chi-squared distribution with one degree of freedom, while their impinging wavefronts have array steering vectors as in (6), with the relative phase delay at the reference antenna randomly generated from a uniform distribution in $[0,2 \pi)$. Again, in pitch estimation the hexagon-shaped array with 3-D Unitary ESPRIT is superior to the crossshaped array with 2-D ESPRIT when $r<2$, inferior to the latter when $r>2$, and has comparable performance for $r=2$. While for roll estimation, the hexagon-shaped array with 3-D Unitary ESPRIT outperforms the cross-shaped array with 2-D ESPRIT in a wider range of body-length-to-wing-breadth ratios up to $r=4$. The same phenomenon is observed for other number of multipaths. 


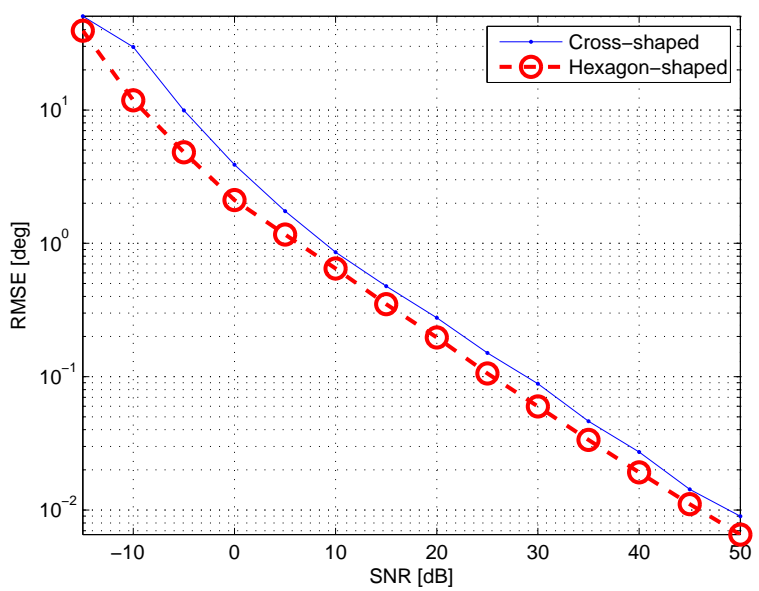

(a) $r=d_{\text {body }} / b_{\text {wing }}=1.25$

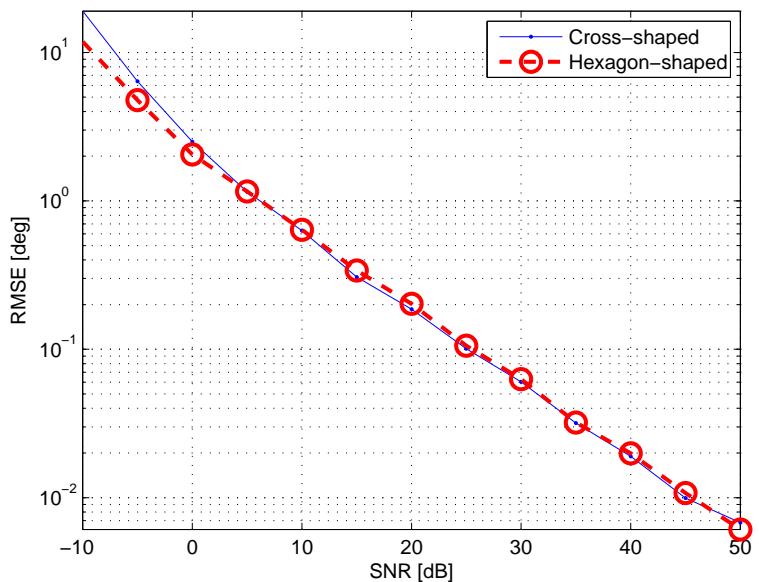

(c) $r=d_{\text {body }} / b_{\text {wing }}=2$

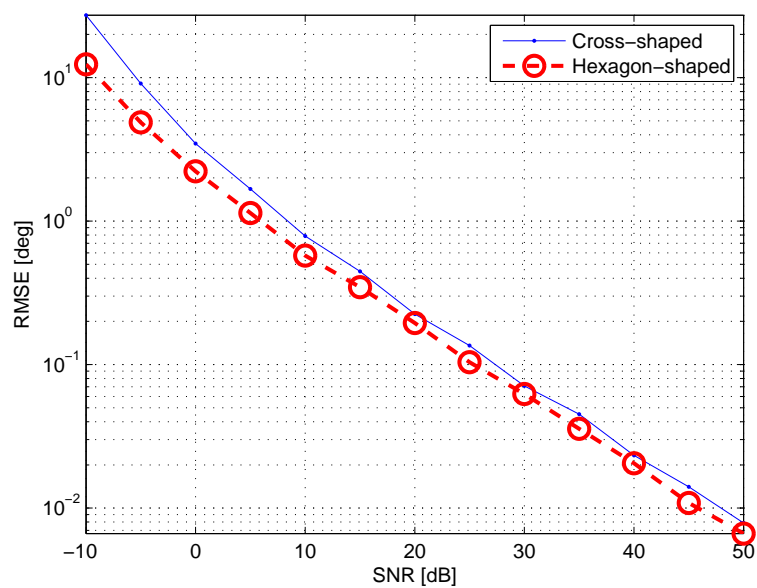

(b) $r=d_{\text {body }} / b_{\text {wing }}=1.5$

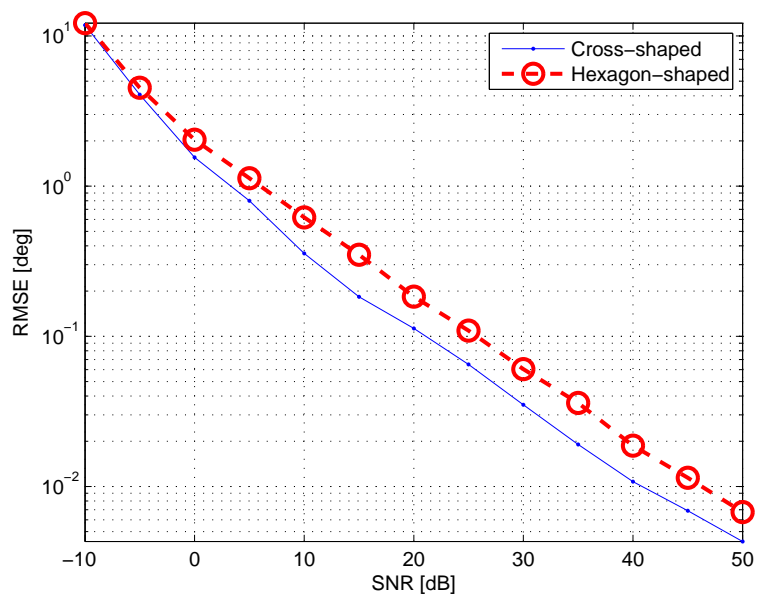

(d) $r=d_{\text {body }} / b_{\text {wing }}=4$

Fig. 7. Comparison of pitch RMSE versus SNR for two attitude estimation schemes: cross-shaped array with 2-D ESPRIT and hexagonshaped array with 3-D Unitary ESPRIT at different body-length-to-wing-breadth ratios.

\section{B. Estimation Performance Dependency on true Pitch and Roll and Range of Inaccuracy}

In Figure 12, we plot the RMSE of the attitude estimates as a function of pitch and roll. We see that when the pitch $\varphi$ approaches the boundary of $\pm \pi / 2$, the pitch estimation suffers a sharp performance degradation. And when both $\varphi$ and $\theta$ are close to the boundaries, namely, $\varphi \rightarrow \pm \pi / 2$ and $\theta \rightarrow \pm \pi / 2$, the roll estimation also deteriorates drastically. Therefore, to safely use the proposed algorithm, such an inaccurate region should be avoided.

\section{CONCLUSiONS}

In this paper, we have proposed an attitude estimation algorithm for unmanned aerial vehicles (UAVs) based on a hexagon-shaped 7-element electronically steerable parasitic antenna radiator (ESPAR) array. The ESPAR array is well-suited for installment in the UAVs with broad wings but short bodies, which is the typical case for most 


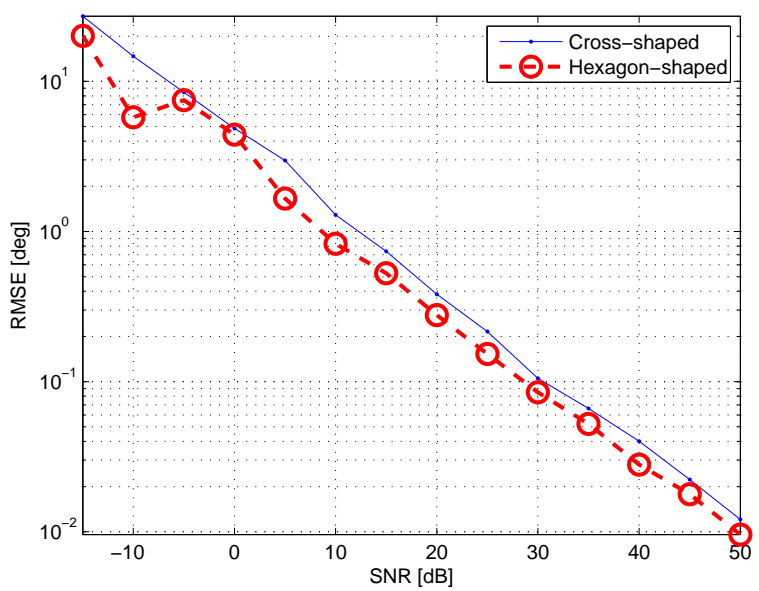

(a) $r=d_{\text {body }} / b_{\text {wing }}=1.25$

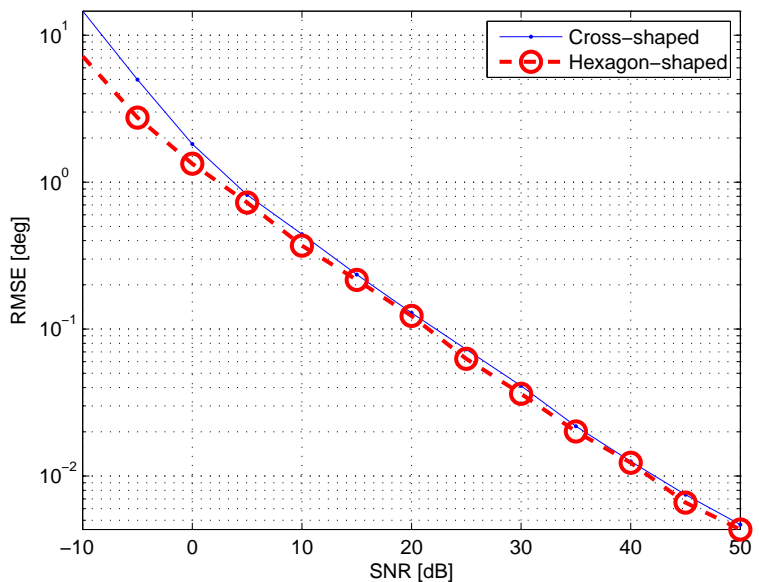

(c) $r=d_{\text {body }} / b_{\text {wing }}=2$

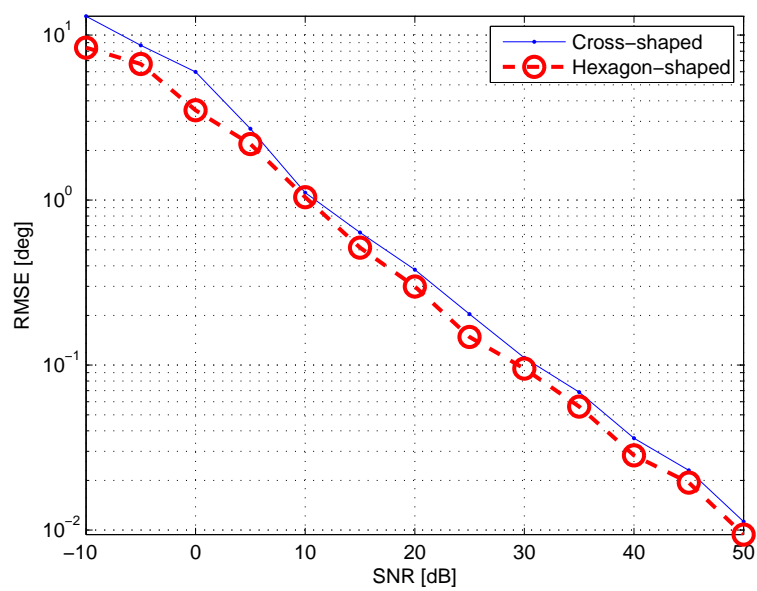

(b) $r=d_{\text {body }} / b_{\text {wing }}=1.5$

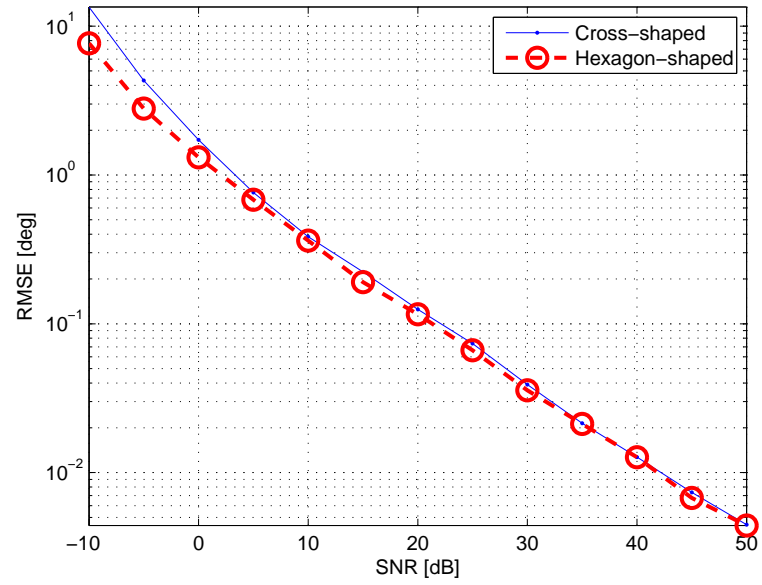

(d) $r=d_{\text {body }} / b_{\text {wing }}=4$

Fig. 8. Comparison of roll RMSE versus SNR for two attitude estimation schemes: cross-shaped array with 2-D ESPRIT and hexagon-shaped array with 3-D Unitary ESPRIT at different body-length-to-wing-breadth ratios.

UAVs. Our proposed solution returns an estimation for the pitch and roll based on the estimates of the phase delays of the line-of-sight path. By exploiting the parallel and centrosymmetric structure of the hexagon-shaped ESPAR array, we apply the 3-dimensional Unitary ESPRIT algorithm for phase delay estimation, which is known for its high estimation accuracy as well as computational efficiency. Then an explicit closed-form formula for the attitude estimates is obtained by solving a system of simultaneous nonlinear equations built based on the geometrical relationship between the UAV attitude and the estimated phase delays.

One limitation of our work is that the attitude estimates depend on the GPS positions which are erroneous. Especially the height is hard to determine and fluctuates largely in practice. It would therefore be interesting to study the sensitivity of the attitude estimates with respect to the GPS positioning errors. As a future work, we intend to use an antenna array being installed at the base station to estimate the UAV position relative to the base 


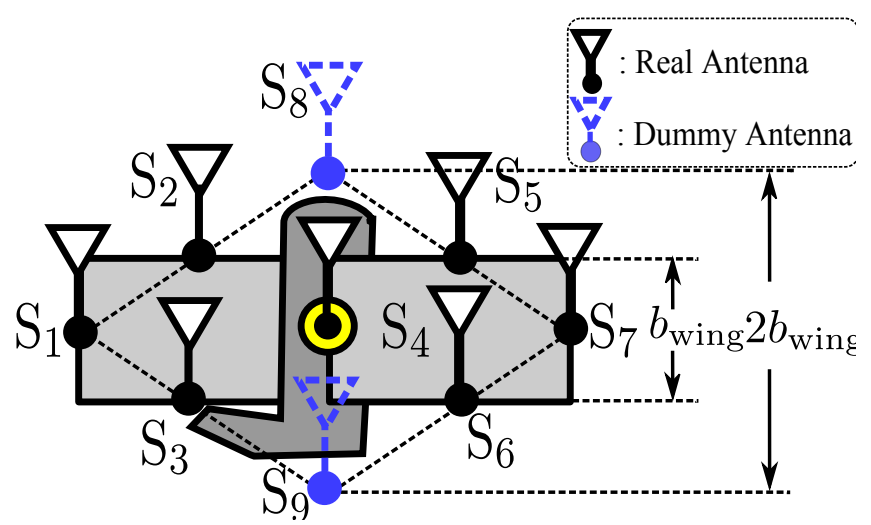

(a) ESPAR array

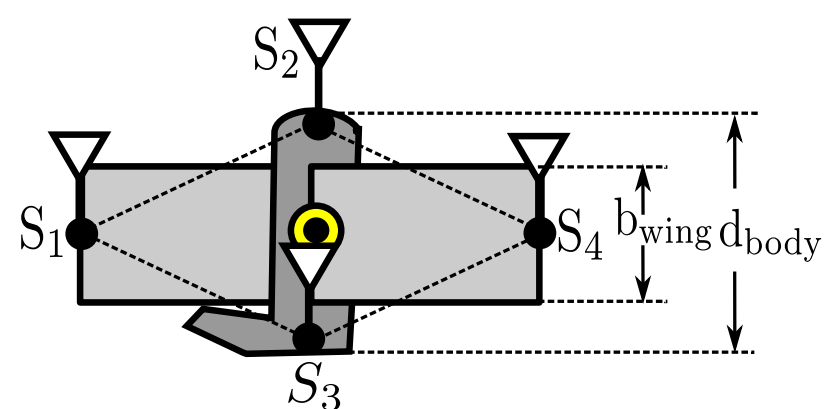

(b) Cross-shaped array

Fig. 9. Array aperture of two kinds of configurations for $r<2$.

station instead of the GPS. In this way, the additional installation of a GPS receiver in UAVs is not required which can further save their costs and weights. Moreover, another future work is to evaluate the proposed scheme using actual flight data. 


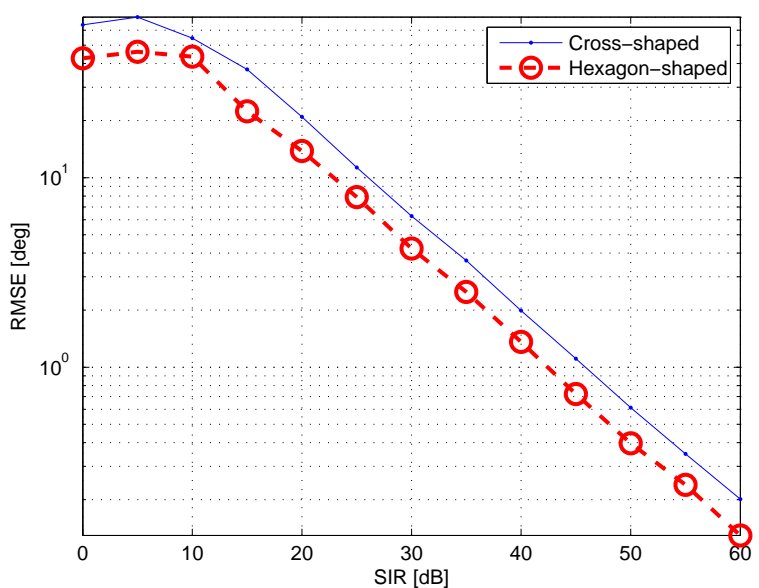

(a) $r=d_{\text {body }} / b_{\text {wing }}=1.25$

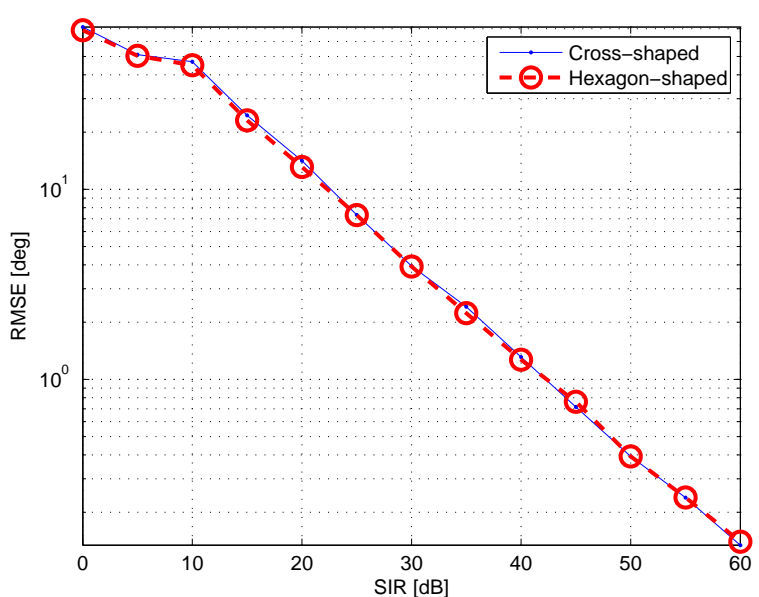

(c) $r=d_{\text {body }} / b_{\text {wing }}=2$

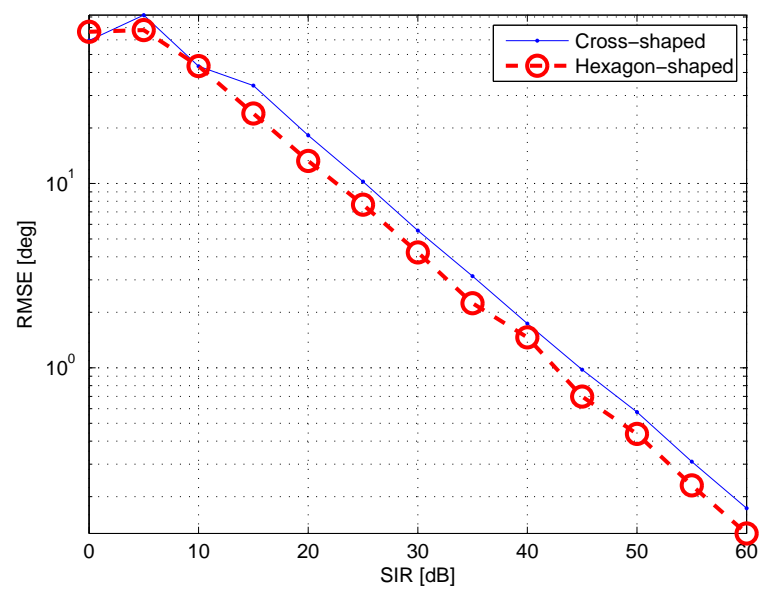

(b) $r=d_{\text {body }} / b_{\text {wing }}=1.5$

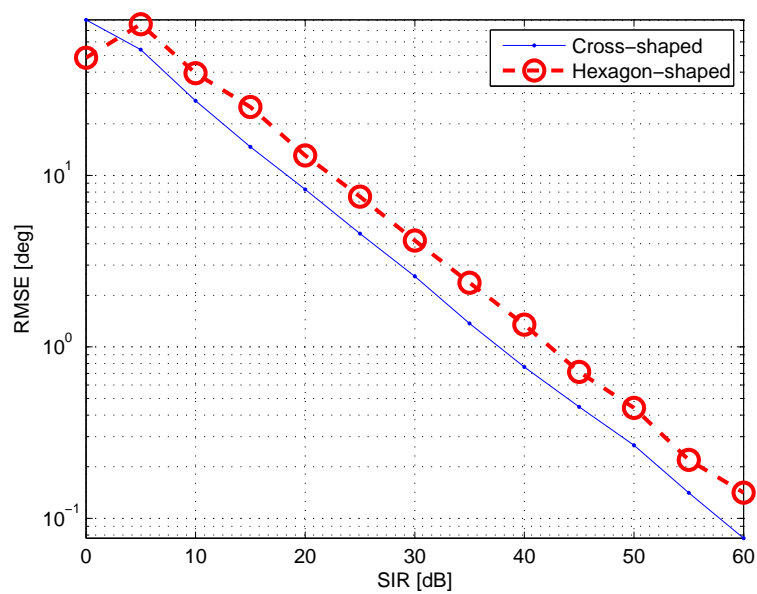

(d) $r=d_{\text {body }} / b_{\text {wing }}=4$

Fig. 10. Comparison of pitch RMSE versus SIR for two attitude estimation schemes: cross-shaped array with 2-D ESPRIT and hexagonshaped array with 3-D Unitary ESPRIT at different body-length-to-wing-breadth ratios. 


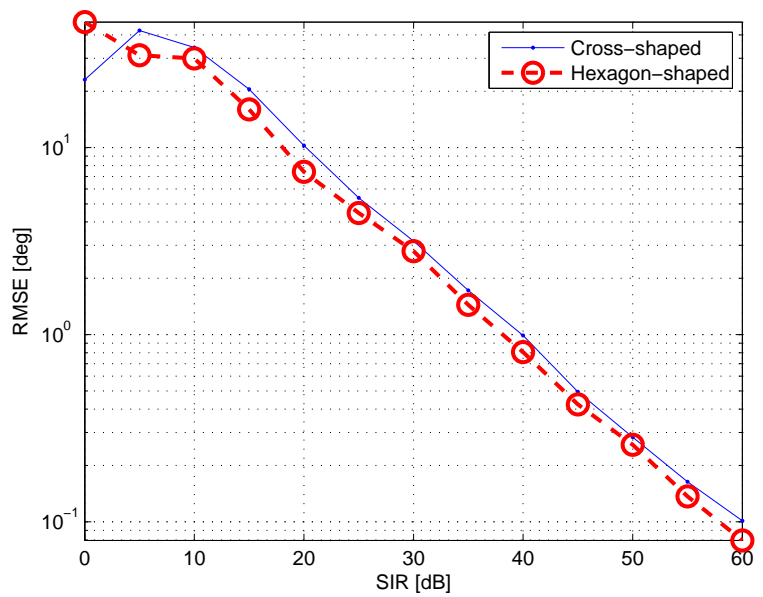

(a) $r=d_{\text {body }} / b_{\text {wing }}=1.25$

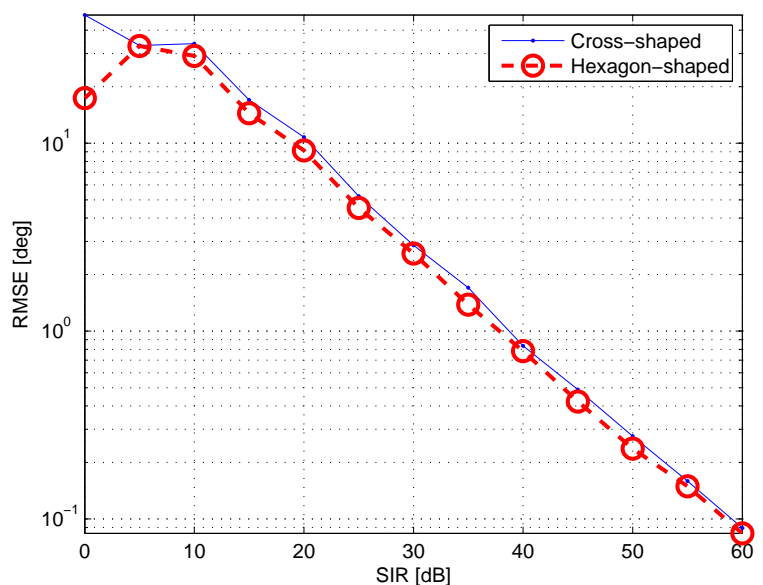

(c) $r=d_{\text {body }} / b_{\text {wing }}=2$

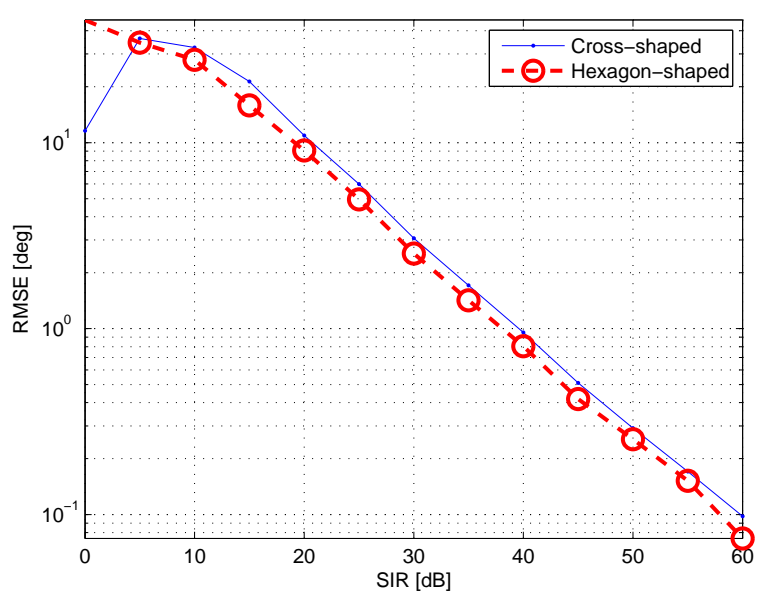

(b) $r=d_{\text {body }} / b_{\text {wing }}=1.5$

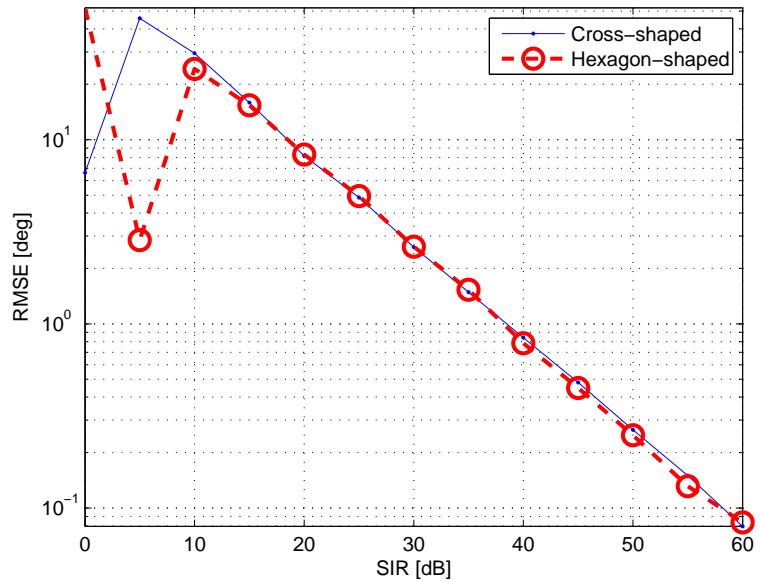

(d) $r=d_{\text {body }} / b_{\text {wing }}=4$

Fig. 11. Comparison of roll RMSE versus SIR for two attitude estimation schemes: cross-shaped array with 2-D ESPRIT and hexagon-shaped array with 3-D Unitary ESPRIT at different body-length-to-wing-breadth ratios. 


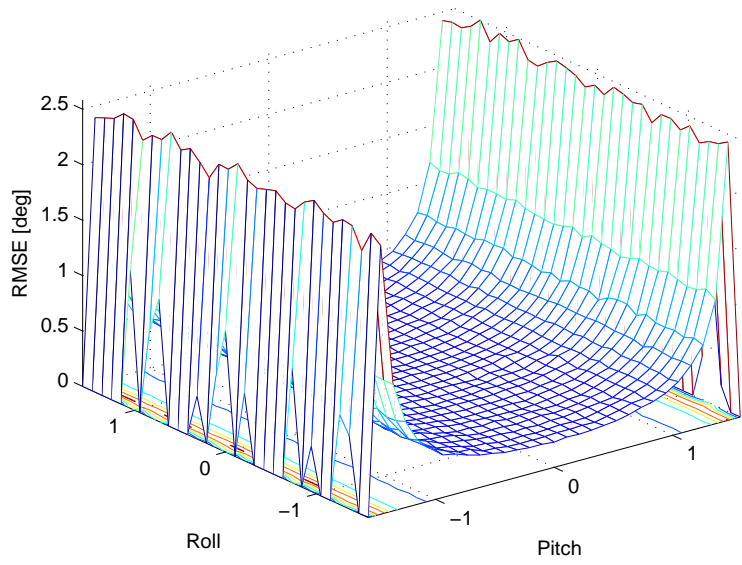

(a) Pitch Estimation

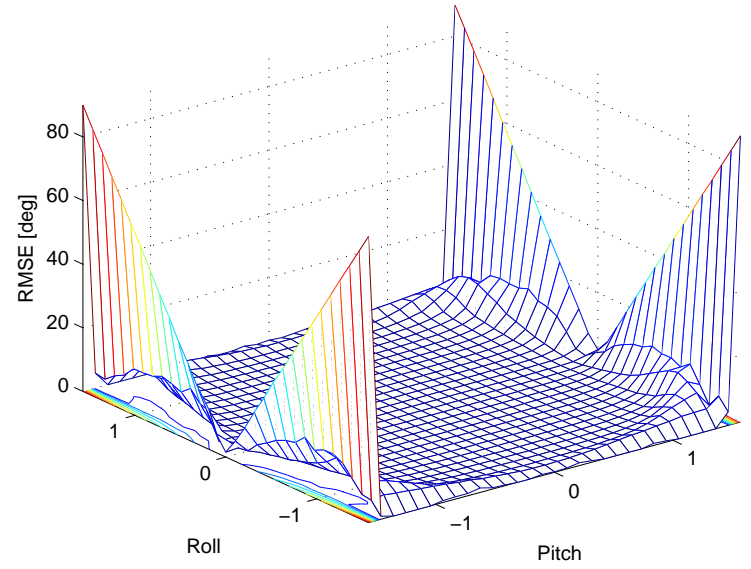

(b) Roll Estimation

Fig. 12. Attitude estimation RMSE as a function of pitch and roll at $\mathrm{SNR}=20 \mathrm{~dB}$. 


\section{APPENDIX A}

SOLVING (30) AND (32)

Solution: Substituting (24) in (29), we obtain

$$
\begin{aligned}
\left\|\boldsymbol{p}_{8}\right\|^{2} & =\left(\boldsymbol{p}_{8}^{\prime}+\boldsymbol{p}_{\mathrm{b}}\right)^{\mathrm{T}}\left(\boldsymbol{p}_{8}^{\prime}+\boldsymbol{p}_{\mathrm{b}}\right)=\left\|\boldsymbol{p}_{8}^{\prime}\right\|^{2}+2 \boldsymbol{p}_{\mathrm{b}}^{\mathrm{T}} \boldsymbol{p}_{8}^{\prime}+\left\|\boldsymbol{p}_{\mathrm{b}}\right\|^{2} \\
& =b_{\text {wing }}^{2}+2\left[\begin{array}{lll}
x_{\mathrm{b}} & y_{\mathrm{b}} & z_{\mathrm{b}}
\end{array}\right] b_{\text {wing }}\left[\begin{array}{c}
-\sin \psi \cos \varphi \\
\cos \psi \cos \varphi \\
\sin \varphi
\end{array}\right]+\left\|\boldsymbol{p}_{\mathrm{b}}\right\|^{2} \\
& =2 b_{\text {wing }}\left[\left(x_{\mathrm{b}} \cos \psi-y_{\mathrm{b}} \sin \psi\right) \cos \varphi+z_{\mathrm{b}} \sin \varphi\right]+b_{\text {wing }}^{2}+\left\|\boldsymbol{p}_{\mathrm{b}}\right\|^{2} \\
& =2 b_{\text {wing }} \sqrt{\left(x_{\mathrm{b}} \cos \psi-y_{\mathrm{b}} \sin \psi\right)^{2}+z_{\mathrm{b}}^{2}} \sin (\varphi+\alpha)+b_{\text {wing }}^{2}+\left\|\boldsymbol{p}_{\mathrm{b}}\right\|^{2} .
\end{aligned}
$$

where

$$
\alpha=\tan ^{-1}\left(\frac{x_{\mathrm{b}} \cos \psi-y_{\mathrm{b}} \sin \psi}{z_{\mathrm{b}}}\right)
$$

According to (30), it holds that

$$
\left\|\boldsymbol{p}_{8}\right\|^{2}=\left(\left\|\boldsymbol{p}_{\mathrm{b}}\right\|+\frac{\lambda}{2 \pi} \hat{\mu}_{\text {body }}\right)^{2}
$$

Substituting (40) in (42) yields

$$
2 b_{\text {wing }} \sin (\varphi+\alpha) \sqrt{\left(x_{\mathrm{b}} \cos \psi-y_{\mathrm{b}} \sin \psi\right)^{2}+z_{\mathrm{b}}^{2}}=\frac{\lambda}{\pi} \hat{\mu}_{\text {body }}\left\|\boldsymbol{p}_{\mathrm{b}}\right\|+\frac{\lambda^{2}}{4 \pi^{2}} \hat{\mu}_{\text {body }}^{2}-b_{\text {wing }}^{2} .
$$

Solving (43) and taking into account that $\varphi \in[-\pi / 2, \pi / 2]$, we obtain the unique solution provided in (31).

Once the pitch estimate $\hat{\varphi}$ is obtained, we compute the roll $\theta$ by (32) in a similar way. Following (29) and (26), we have

$$
\begin{aligned}
&\left\|\boldsymbol{p}_{1}\right\|^{2}=\left(\boldsymbol{p}_{1}^{\prime}+\boldsymbol{p}_{\mathrm{b}}\right)^{\mathrm{T}}\left(\boldsymbol{p}_{1}^{\prime}+\boldsymbol{p}_{\mathrm{b}}\right)=\left\|\boldsymbol{p}_{1}^{\prime}\right\|^{2}+2 \boldsymbol{p}_{\mathrm{b}}^{\mathrm{T}} \boldsymbol{p}_{1}^{\prime}+\left\|\boldsymbol{p}_{\mathrm{b}}\right\|^{2} \\
&=\frac{1}{4} d_{\mathrm{wing}}^{2}+2\left[\begin{array}{lll}
x_{\mathrm{b}} & y_{\mathrm{b}} & z_{\mathrm{b}}
\end{array}\right] \frac{1}{2} d_{\text {wing }}\left[\begin{array}{ccc}
\cos \hat{\varphi} & -\sin \psi \cos \hat{\varphi} & \sin \psi \sin \hat{\varphi} \\
\sin \hat{\varphi} & -\cos \psi \cos \hat{\varphi} & -\cos \psi \sin \hat{\varphi} \\
0 & \sin \hat{\varphi} & \cos \hat{\varphi}
\end{array}\right]\left[\begin{array}{c}
\cos \theta \\
0 \\
\sin \theta
\end{array}\right] \\
&=d_{\mathrm{wing}}\left[\left(x_{\mathrm{b}} \cos \psi+y_{\mathrm{b}} \sin \psi\right) \cos \theta+\left(x_{\mathrm{b}} \sin \psi \sin \hat{\varphi}-y_{\mathrm{b}} \cos \psi \sin \hat{\varphi}+z_{\mathrm{b}} \cos \hat{\varphi}\right) \sin \theta\right]+ \\
& \frac{1}{4} d_{\text {wing }}^{2}+\left\|\boldsymbol{p}_{\mathrm{b}}\right\|^{2} \\
&=d_{\mathrm{wing}} \sin (\theta+\beta) \sqrt{\left(x_{\mathrm{b}} \cos \psi+y_{\mathrm{b}} \sin \psi\right)^{2}+\left(x_{\mathrm{b}} \sin \psi \sin \hat{\varphi}-y_{\mathrm{b}} \cos \psi \sin \hat{\varphi}+z_{\mathrm{b}} \cos \hat{\varphi}\right)^{2}}+ \\
& \frac{1}{4} d_{\mathrm{wing}}^{2}+\left\|\boldsymbol{p}_{\mathrm{b}}\right\|^{2} .
\end{aligned}
$$

where

$$
\beta=\tan ^{-1}\left(\frac{x_{\mathrm{b}} \cos \psi+y_{\mathrm{b}} \sin \psi}{x_{\mathrm{b}} \sin \psi \sin \hat{\varphi}-y_{\mathrm{b}} \cos \psi \sin \hat{\varphi}+z_{\mathrm{b}} \cos \hat{\varphi}}\right) .
$$

From (32), it follows that

$$
\left\|\boldsymbol{p}_{1}\right\|^{2}=\left(\left\|\boldsymbol{p}_{\mathrm{b}}\right\|+\frac{\lambda}{2 \pi} \hat{\mu}_{\text {wing }}\right)^{2}
$$


Substituting (44) in (46), we obtain

$$
\begin{aligned}
& d_{\text {wing }} \sin (\theta+\beta) \sqrt{\left(x_{\mathrm{b}} \cos \psi+y_{\mathrm{b}} \sin \psi\right)^{2}+\left(x_{\mathrm{b}} \sin \psi \sin \hat{\varphi}-y_{\mathrm{b}} \cos \psi \sin \hat{\varphi}+z_{\mathrm{b}} \cos \hat{\varphi}\right)^{2}} \\
& =\frac{\lambda}{\pi} \hat{\mu}_{\text {wing }}\left\|\boldsymbol{p}_{\mathrm{b}}\right\|+\frac{\lambda^{2}}{4 \pi^{2}} \hat{\mu}_{\text {wing }}^{2}-\frac{1}{4} d_{\text {wing }}^{2} .
\end{aligned}
$$

Solving (47) yields (33) and (34).

\section{REFERENCES}

[1] J. P. C. L. da Costa, S. Schwarz, L. F. de A. Gadêlha, H. C. de Moura, G. A. Borges, and L. A. R. Pinheiro, "Attitude determination for unmanned aerial vehicles via an antenna array," in Proc. ITG IEEE Workshop on Smart Antennas (WSA'12), Dresden Germany, Mar. 2012.

[2] G. L. Sitzmann and G. H. Drescher, "Tactical ballistic missiles trajectory state and error covariance propagation," in Proc. IEEE Position Location and Navigation Symposium, Las Vegas, NV, USA, Apr. 1994, pp. 839-844.

[3] K. Liu, J. P. C. L. da Costa, H. C. So, L. F. A. Gadelha, and G. A. Borges, "Improved attitude determination for unmanned aerial vehicles with a cross-shaped antenna array," in Proc. 14th IASTED International Conference on Signal and Image Processing (SIP'2012), Honolulu, Hawaii, USA, Aug. 2012, pp. 60-67.

[4] M. S. Sharawi, O. A. Rawashdeh, and D. N. Aloi, "Performance of an embedded monopole antenna array in a UAV wing structure," in Proc. of 15th IEEE Mediterranean Electrotechnical Conference (MELECON 2010), Valetta, Malta, Apr. 2010, pp. 835-838.

[5] C. Plapous, J. Cheng, E. Taillefer, A. Hirata, and T. Ohira, "Reactance domain MUSIC algorithm for ESPAR antennas," in Proc. 33rd European Microwave Conference EuMW2003, Munich, Germany, Oct. 2003, pp. 793-796.

[6] E. Taillefer, E. Chu, and T. Ohira, "ESPRIT algorithm for a seven-element regular-hexagonal shaped ESPAR antenna," in Proc. European Conference on Wireless Technology (ECWT 2004), Amsterdam, Netherlands, Oct. 2004.

[7] F. Roemer and M. Haardt, "Using 3-D Unitary ESPRIT on a hexagonal shaped ESPAR antenna for 1-D and 2-D direction of arrival estimation," in Proc. International ITG/IEEE Workshop on Smart Antennas (WSA'05), Duisburg, Germany, Apr. 2005.

[8] P.-J. Park, S.-M. Choi, D.-H. Lee, and B.-S. Lee, "Performance of UAV(unmanned aerial vehicle) communication system adapting WiBro with array antenna," in Proc. of 11th International Conference on Advanced Communication Technology (ICACT 2009), South Korea, Feb. 2009, vol. 2, pp. 1233-1237.

[9] Yohandri V. Wissan, I. Firmansyah, P. R. Akbar, J. T. Sri Sumantyo, and H. Ku, "Development of circularly polarized array antenna for synthetic aperture radar sensor installed on UAV," Progress In Electromagnetics Research C, vol. 19, pp. 119-133, 2011.

[10] C. Cohen, Attitude Determination using GPS, Ph.D. thesis, Department of Aeronautics \& Astronautics, Stanford University, CA, USA, 1992.

[11] J. Jang, S. Lee, and C. Kee, "Performance enhancement of attitude determination system by combining single and multiple antennas," in Proc. of the 17th International Technical Meeting of the Satellite Division of The Institute of Navigation (ION GNSS 2004), Long Beach, CA, September 2004, pp. 2066-2073.

[12] M. Haardt, F. Roemer, and G. Del Galdo, "Higher-order SVD based subspace estimation to improve the parameter estimation accuracy in multi-dimensional harmonic retrieval problems," IEEE Transactions on Signal Processing, vol. 56, no. 7, pp. 3198 - 3213, July 2008.

[13] Y.C. Lai and S.S. Jan, "Attitude estimation based on fusion of gyroscopes and single antenna GPS for small UAVs under the influence of vibration," GPS Solutions, vol. 15, no. 1, pp. 67-77, 2010.

[14] M. Haardt, Efficient One-, Two-, and Multidimensional High-Resolution Array Signal Processing, 3-8265-2220-6. Shaker Verlag, Aachen, Germany, 1996.

[15] A. Lee, "Centrohermitian and skew-centrohermitian matrices," Linear Algebra and its Applications, vol. 29, pp. 205-210, 1980. 